l'opération s'achève au four Héroult, dans lequel le métal séjourne de deux heures à deux heures et demie. Cela semble d'autant plus long,que la déphosphoration est déjà très poussée au four Welmann. En effet, les éprouvettes, prélevées dians la poche, au moment de la coulée dans le four électrique, accusent pour la première coulée 0,03 I et pour la deuxième coulée 0,014 de $\mathrm{Ph}$. Le four Héroult ne fait que désulfurer et perfectionner la déphosphoration. Les résultats obtenus à ce four sont remarquables. Les chiffres relevés sur les livres de l'usine de Remscheid indiquent des teneurs on $\mathrm{S}$ et $\mathrm{Ph}$ dépassant 0,10 pour Ioo.

L'usine de Remscheid fait, d'ailleurs, des aciers au creuset depuis de longues années; aussi le four Héroult, desservi par un personnel comnaissant à fond la fabrication de l'acier, s'y trouve-t-il dans des conditions exceptionnellement bonnes.

Au four Girod, à Ugine, au cours de cinq coulées faites avec de lia fonte de Chasse, à 0,046 de $\mathrm{Ph}$, et des riblons à 0,075 , on a obtenu des aciers dont la teneur en $\mathrm{Ph}$ varie de 0,017 à 0,048 et le $\mathrm{S}$ de 0,013 à. 0,035 . Il semble, au premier abord, qu'il n'y ait là qu'une épuration courante que l'on peut obtenir au four Martin. Mais il faut observer que les cinq opérations au four Girod ont été faites sans aucune précaution, et avec une durée variant de trois heures un quart à cinq hetres, pour une production de I 000 à I $400 \mathrm{kgs}$, en partant d'une charge froide et cela par un personnel n'ayant jamais vu fabriquer d'acier. On se rend facilement compte que ce même four, mis entre les mains d'aciéristes expérimentés, donnerait des résultats au moins aussi brillants, au point de vue chimique, que le four Héroult. D'ailleurs, sur trie quinzaine de coulées relevées au hasard sur le livre du laboratoire de cette usine, et comprenant toutes les nuances d'acier, on trouve les proportions suivantes : $\mathrm{S}=0,001$ à $0,035, \mathrm{Ph}=$ traces à 0,037 . Ces coulées ont été faites dans des conditions très mauvaises comme personnel et matériel, en présence des diverses délégations venues à Ugine pour étudier le four, c'est-àdire dans des conditions défavorables pour une étude suivie. Cette étude va être rationnellement entreprise, et il est à süpposer que, sous peu, l'usine d'Ugine donnera d'une façon suivie des aciers de toutes qualités, et d'une pureté exceptionnelle.

Comparant maintenant ces cleux fours au point de vue installation et exploitation, M. Saconney constate les avantages et inconvénients suivants :

Avantage du four Girod, parce qu'il n'a qu'une seule électrode ou plutôt parce que, lorsqu'il y en a plusieurs, elles sont en parallèle, donc pas de courts-circuits à craindre par l'enveloppe, cela permet de réduire le jeu entre l'électrode et le trou servant à son passagie dans le couvercle, d'où moins de perte de chaleur, moins d'usure dans les électrodes. A cause du faible voltage, toute difficulté est supprimée au point de vue de l'isolement et la mise en marche est beaucoup plus facile et plus rapide. Pas de court-circuit à craindre par la charge elle-même lorsque l'on charge en matières solides, ce qui rend très irrégulier et même dangereux le départ sur riblons froids au four Héroult.

Le réglage, à Remscheid, même en chargeant l'acier liquide, doit être fait à la main pendant une demi-heure à trois quarts d'heure, avant de le faire automatiquement.

Avantage du four Héroult dû à la plus faible intensité. L'intensité double du four Girod oblige à doubler la dépense pour les conducteurs; c'est une augmentation du prix de premicr établissement, mais cette augmentation est largement compensée, d'autre part, comme on va le voir.

Avantage du four Girod sur le dispositif de suspension et réglage des électrades, plus simple et plus pratique permettant d'isoler dans la salle des machines les appareils délicats de réglage.

Le four Girod utilise mieux l'énergie calorifique développée par la résistance, car dans ce four la résistance intéresse la totalité de la charge, tandis que dans le four Héroult èlle n'intéresse que les couches stpérieures, le fond doit être réchauffé par conductibilité. Oń a constaté à La Praz qu'il fallait plus de deux heures pour arriver à chauffer le fond. Ceci expliquerait le long séjour du bain dans le four électrique constaté a Remscheid.

L'avantage signalé plus haut pour le four Héroult est très relatif, car il est détruit largement par le prix plus élevé de ce four.

Le four Héroult coûte environ 30.000 francs, y compris les appareils de réglage, sans électrodes ni fondations.

Le four Girod coûte environ 20000 francs dans les mêmes conditions, soit donc en faveur de ce dernier une économie de plus de 10000 francs compensant et au delà la plus forte dépense pour les conducteurs.

Comme conclusion, les avantages $d u$ four Héroult ne portent que sur la première installation; ceux du four Girod, qui sont indéniables, se font sentir d'une façon constante pendant toute la durée de la marche du four, de sorte que M. Saconney miontre une préférence marquée pour le four Girod, qui serait d'un rendement plus économique, tout en donnant, comme pour le four Héroult, toutes les qualités d'acier désirées.

M. Stassano fait observer, en réponse aux remarques de M. Saconney, que celui-ci a oublié de dire que les deux premières coulées faites en sa présence ne doivent pas être retenues, une erreur ayant été commise dans le calcul des dosages de ces deux premières charges, comme il le lui fit remarquer immédiatement. Quant aux autres opérations, elles constituaient chacune un premier essai avec les différentes charges employées. Or, comme on l'a vu, on dioit, dans le four électrique, arriver au produit final avec dosage fait au préalable, parce que cela répond parfaitement à la théorie et à la pratique de cet appareil, mais on ne peut pas prétendre atteindre ce but avec un simple calcul théorique, sans le contrôle d'un essai pratique. Si une telle manière de procéder était réalisable, le four électrique constituerait un appareil donniant exactement les résultats théoriques, ce qu'on ne peut raisonnablement demander.

\section{Recherches sur l'influence de la disposition des Électrodes dans les Prises de terpe industrielles}

L'étude théorique, objet de cet article, a été faite pour un transport d'énergie électrique par le système continu-série. On avait envisagé dans ce transport le retour par la terre. Il s'agissait d'établir d'avance la répartition, la forme et les dimensions des prises de terre, pour une puissance déterminée, et pour les pertes admises par l'économie du projet et par l'Administration compétente.

Soit en un point $O$, situé dans un milieu homogène indéfini, une quantité d'électricité définie et constante $A$.

Il se produit autour du point $O$ un champ électrique; soit $p$ la distance au point $O$ d'un point quelconque $M$.

Le potentiel $U$, créé par $A$, est en ce point : $U=\frac{A}{p}$

L'équation d'une surface équipotentielle est :

$$
U=\text { Constante d’où } \rho=\text { Constante }
$$

Dans ce cas, les surfaces équipotentielle sont donc des sphères.

D'autre part, la surface d'une électrode est une surface que nous supposons maintenue au même potentiel. Il suffit pour cela que l'électrode soit de conductibilité infinie, ou pratiquement très grande par rapport à la conductibilité du milieu homogène dans lequel elle est plongée. 
La surface de l'électrode est done, pratiquement, une surface équipotentielle, et la charge $A$ se répartira à la surface de l'électrode de telle sorte que :

$$
\int \frac{\mathrm{d} A}{\mathrm{p}}=\text { Constante }
$$

pour tous les points de la surface de l'électrode, et de l'électrode entière si elle est homogène.

Si nous supposons que l'électrode soit sphérique, ce résultat sera obtenu par une répartition uniforme de la charge $A$ à la surface de l'électrode, et l'on sait que, dans ce cas, on peut considérer que toute la charge est concentrée au centre de la sphère.

Supposons donc que l'électrode soit sphérique et de rayon $r$. Le potentiel produit $\mathrm{cn}$ un point distant de $\rho \mathrm{du}$ centre $(p>r)$, sera:

$$
V=\frac{A}{\rho}
$$

et le champ en ce point sera : $\quad H=-\frac{\mathrm{d} V}{\mathrm{~d}_{p}}=\frac{A}{p^{2}}$

Soit $C$ la conductibilité du milieu homogène.

L'intensité du courant produit par le champ $H$ sera :

$$
I=C S H=4 \pi C_{p}^{2} \frac{A}{\rho^{2}}=4 \pi C A=4 \pi C V_{\rho}
$$

d'où :

$$
V=\frac{I}{4 \pi C_{p}}
$$

Si nous supposons le milieu homogène indéfini limité par un plan passant par le centre de la sphère, celle-ci sera réduite à une hémisphère, et nous aurons :

$$
S=2 \pi q^{2} \quad \text { et : } \quad V=\frac{I}{2 \pi C_{p}}
$$

En particulier, à la surface de l'électrode, on aura :

$$
V=\frac{I}{2 \pi C r}
$$

$V$ sera le potentiel auquel il faudra maintenir l'électrode pour diffuser, en quelque sorte, le courant $I$ dans la terre.

En réalité, la charge n'est pas uniformément répartie à la surface d'une demi-sphère conductrice, mais une charge. concentrée en un point de la surface du demi-milieu indéfini donnera un champ dont les surfaces équipotentielles seront des hémisphères. Soient $V_{1}$ et $V_{2}$ les potentiels de deux électrodes, et $r_{1}$ et $r_{2}$ leurs rayons. Elles ont toutes deux à diffuser le même courant $I$, mais en sens inverse. On a donc :

$$
V_{1}=\frac{I}{2 \pi C r_{1}} \quad V_{\mathrm{a}}=\frac{-I}{2 \pi L r_{2}}
$$

et : $\quad V_{1}-r_{2}=\frac{I}{2 \pi C} \times\left(\frac{1}{r_{1}}+\frac{1}{r_{2}}\right)=\frac{I}{2 \pi C} \times \frac{r_{1}+r_{0}}{r_{1} \times r_{2}}$

Si nous faisons $r_{1}=r_{2}=r$, nous retombons sur la formule $V_{1}-V_{\mathrm{g}}=\frac{I}{\pi \cdot r}$.

Nous pouvons maintenant calculer les dimensions d'une électrode devant diffuser un courant, $I=200$ ampères, dans un sol de conductibilité moyenne, $C=\frac{1}{5000}$ microhm-cen- timètre, avec une chute de tension $V=$ I 00 volts par prise, ou 200 volts pour le retour complet par la terre.

La formule : $\quad V=\frac{I}{2 \pi C r}$ donne : $r=\frac{I}{2 \pi C V}=\frac{200}{2 \pi \frac{1}{5000} 100}=1600 \mathrm{~cm}$.

c'est-à-dire : $r=16$ mètres.

La formule ci-dessus semble montrer que le même potentiel $V$ suffira pour faire débiter le courant $I$ par $n$ électrodes de rayon $\frac{r}{n}$ et débitant $\frac{I}{n}$, par exemple to électrodes de Im6o débitant 10 ampères.

Voyons si cela se vérifie.

Nous avons supposé jusqu'ici que la charge $A$ était concentrée sur un seul point. Si nous la subdivisons en $n$ électrodes, son influence ne sera plus la même. Nous allons examiner les deux cas les plus simples, et qui se rapprochent le plus des dispositions que l'on sera amené à adopter :

$\mathrm{I}^{\circ}$ La prise de terre se compose de $n$ électrodes réparties uniformément sur une circonférence de rayon $R$ et de centre $\mathrm{O}$;

$2^{\circ}$ La prise se compose de $n$ électrodes réparties uniformément sur une droite de longueur $L$.

Io Electrodes disposées circulairement. - La répártition de la charge étant faite d'une façon uniforme, chacune des $n$ électrodes aura une charge $: a=\frac{A}{n}$.

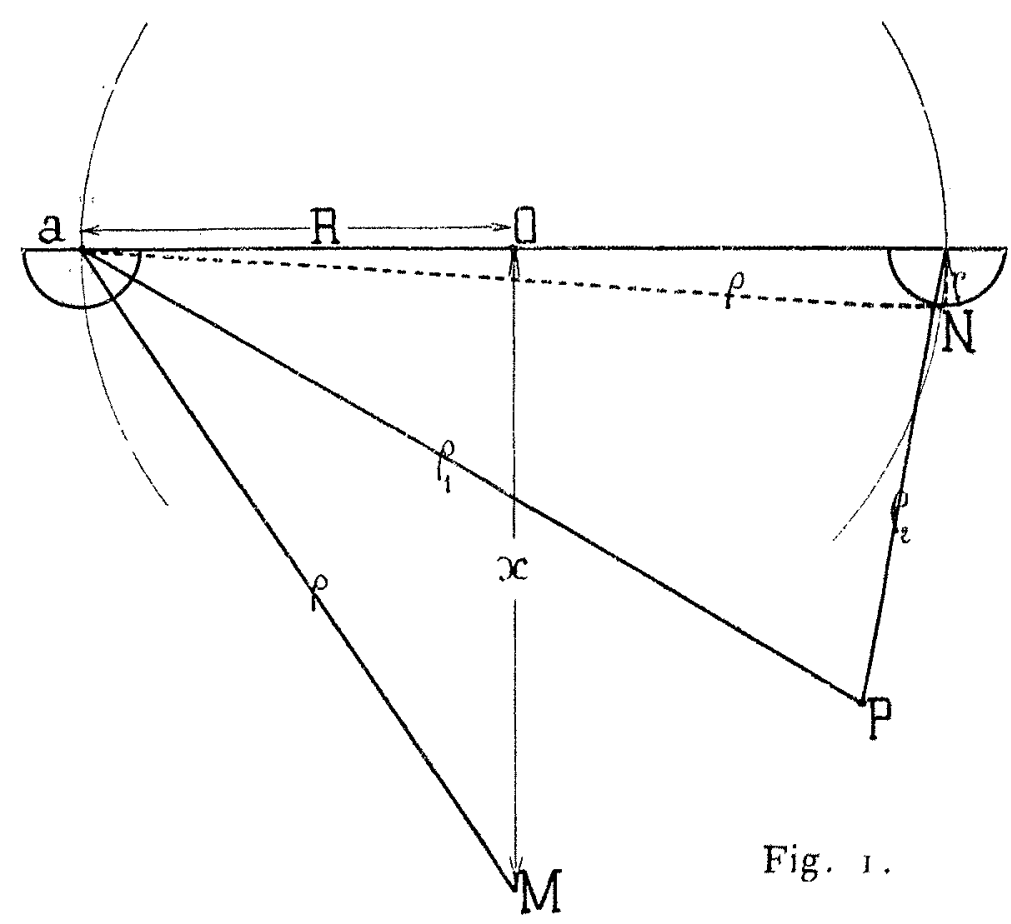

Le potentiel créé en un point $\mathrm{P}$ quelconque de l'espace sera:

$$
V=a \mathrm{\Sigma} \frac{\mathrm{I}}{\mathrm{p}}
$$

Considérons d'abord un groupe de 2 électrodes, et pre- 
nons un point $M$, sur la perpendiculaire au milieu de la droite qui les joint, à une distance $x$ du centre $O$.

$$
V=a \mathrm{i} \frac{\mathrm{I}}{\mathrm{p}}=2 \frac{a}{\mathrm{P}}
$$

or : $\quad p=\sqrt{R^{2}+x^{2}} \quad V=\frac{2 a}{\sqrt{R^{2}+x^{2}}}$

Dans le sens perpendiculaire au premier :

$$
\begin{gathered}
\Sigma \frac{1}{p}=\frac{1}{x+R}+\frac{1}{x-R}=\frac{2 x}{x^{2}-R^{2}} \\
Y^{\prime}=\frac{2 a x}{x^{2}-R^{2}}
\end{gathered}
$$

On voit que lorsque $x$ augmente, et que $R$ devient négligeable par rapport à $x$, ces deux valeurs de $V$ tendront à devenir :

$$
V=\frac{2 a}{x}
$$

Il en serait de même pour un nombre quelconque d'électrodes réparties uniformément sur une circonférence de centre $\mathrm{O}$.

Lorsqu'on s'éloigne de la prise de terre, on trouve donc des surfaces équipotentielles se rapprochant de plus en plus de la sphère. Nous retombons sur le cas déjà étudié, è nous avons :

$$
\begin{gathered}
H=\frac{a n}{x^{2}} \quad \text { et } \quad I=C S H={ }_{2} C \pi x^{2} \frac{a n}{x^{2}}=2 \pi C a n \\
V=\frac{a n}{x}=\frac{I}{2 \pi C x} \quad \text { d'où }: \quad I=2 \pi C V x
\end{gathered}
$$

Nous arrivons donc à cette conclusion que, à une distance suffisante de la prise de terre, c'est-à-dire de l'ensemble des électrodes, la forme et la disposition de celles-çi n'interviennent plus, et tout se passe comme si $\mathrm{cn}$ avait une électrode unique émettant le courant total.

Si nous voulons avoir en un point lá chute linéaire de tension, elle nous sera donnée par la dérivée du potentiel par rapport à la distance, soit :

$$
H=-\frac{\mathrm{d} V}{\mathrm{~d}_{p}}=\frac{I}{\operatorname{CSS}^{\prime}}=\frac{I}{2 \pi C_{q^{2}}}
$$

Si nous faisons : $I=200$, et $C=\frac{1}{5000}$ il faut, pour que la chute de tension kilométrique soit de 2 volts, ou $\mathrm{O}^{\mathrm{v}}, 00002$ par centimètre, que :

$$
\hat{p}^{2}=\frac{200}{2 \pi \times 0,00002 \times \frac{1}{5000}}=\frac{10^{11}}{12,50}
$$

d'où : $\quad \rho=89400 \mathrm{~cm} .=894$ mètres.

Il faudra donc que les prises de terre soient au moins à $900 \mathrm{~m}$. des terres de l'Administration. Il est à remarquer toutefois que les terres de parafoudre, pour postes téléphoniques par exemple, peuvent, sans aucun inconvénient, se truuver beaucoup plus prés, puisqu'elles sont, en temps normal, isolées du système électrique infuençable, et qu'elles ne lui sont reliées que pendant un coup de foudre.
A cette distance de $900 \mathrm{~m}$. le potentiel est :

$$
V=\frac{A}{\rho}=\rho H
$$

soit a volt 8 .

Si nous respectons toujours cette condition, la différence de tension maxima à laquelle pourra être soumise uné ligne de l'Etat sera 3 volts 6.

Remarquons, en passant, que, pour que la protection soit efficace, il faudra que, non seulement les terres, mais encore les lignes de l'Administration soient hors de la zone dangereuse, car la rupture d'un isolateur produit une terre, et cela arrive très fréquemment. 11 est vrai que, pour les lignes, on peut adimetre une marge plus grande, car ces terres accidentelles sont rarement franches.

Pour éviter les phénomènes d'électrolyse, on devra également tenir les conduites métalliques des villes hors de la zone dangëreuse.

Reprenons l'étude des surfaces équipotentielles. Lorsque $p<R$, la distance $d$ entre deux électrodes est :

$$
d=\frac{2 \pi R}{n}
$$

Considérons un point $\mathrm{N}$ voisin d'un des points d'émission, tel que $r<\frac{d}{2}, n$ étant notable, $r$ sera petit devant $R$.

Le potentiel seia en $\mathrm{N}$ :

$$
V=\frac{a}{r}+a \Sigma \frac{\mathbf{I}}{\rho}
$$

En prenant pour $q$ une valeur moyenne $R_{m}$, nous aurons:

$$
V=\frac{a}{r}+a(n-1) \frac{1}{R_{m}}
$$

La suface équipotentielle n'est plus une sphère, cependant, si $R_{m}$ est très grand vis-à-vis de $r$, nous pourrons la considérer comme telle, et elle aura $r$ comme rayon.

Comme on le voit, le potentiel en un point se compose de deux parties : l'une est donnée par le point d'émission considéré, et l'autre par l'ensemble des autres points. Nous pouvons les désigner par $V^{\prime}$ et $V^{\prime}$.

$$
V^{\prime}=V^{\prime}+V^{\prime}
$$

De même, le champ en ce point sera :

$$
\begin{aligned}
& H=-\frac{\mathrm{d} V}{\mathrm{~d} x}=-\frac{\mathrm{d} V^{\prime}}{\mathrm{d} r}-\frac{\mathrm{d} V^{\prime \prime}}{\mathrm{d} \frac{\mathrm{l}}{\mathrm{\Sigma} \frac{\mathrm{I}}{\rho}}} \\
& \text { pour l'intensité. }
\end{aligned}
$$

L'intensité $i$ émise par le centre considéré sera celle qui correspond au premier terme, et le $2^{\mathrm{e}}$ terme représentera le courant, parti des autres centres d'émission, et passant au paint étudié. On a done:

$$
\begin{gathered}
H=-\frac{\mathrm{d} V}{\mathrm{~d} r}=\frac{a}{r^{2}} \\
i=C S H=2 \pi C r^{\circ} \cdot \frac{a}{r^{2}}=2 \pi C a
\end{gathered}
$$


d’où : $\quad a=\frac{i}{2 \pi C} \quad$ et $\quad V=\frac{i}{2 \pi C r}+\frac{(n-1) i}{2 \pi C R_{m}}$

Le courant total serait : $\quad I=n i, \quad$ d'où : $\quad i=\frac{I}{n}$ et, en remplaçant, on a :

$$
V=\frac{I}{2 \pi C n r}+\frac{(n-1) I}{2 \pi C n R_{m}}
$$

Donc, si l'on compare une prise de terre formée d'une seule électrode hémisphérique de rayon $n r$, à une prise formée de $n$ électrodes hémisphériques de rayon $r$, répartie uniformément suivant une circonférence, nous avons:

dans un cas :

$$
V_{0}=\frac{I}{2 \pi C n r}
$$

et dans l'autre : $\quad V=\frac{I}{2 \pi C n r}+\frac{(n-1) I}{2 \pi C n R_{m}}$

si donc on a : $\quad R_{m}=(n-\mathrm{I}) r$

on aura :

$$
V=2 V_{0}
$$

$\frac{n-1}{R_{m}}$ n'est pas autre chose que $\Sigma \frac{1}{p}$, pour lequel on a pris une valeur approximative moyenne. Si nous prenons : $n=10$, on trouve en faisant l'épure :

$$
\Sigma \frac{1}{\rho}=\frac{n-1}{1,15 R}=\frac{7.8}{R}
$$

$R$ étant le rayon de la circonférence sur laquelle sont les électrodes.

De sorte que si : $R=0,78 n r$ et si : $\quad R=n r$

$$
\begin{aligned}
& V=2 V_{0} \\
& V=1,78 V_{0}
\end{aligned}
$$

Si nous voulons que le supplément de chute de tension ne dépasse pas $\frac{V_{0}}{10}$, nous devrons avoir :

$$
\frac{1}{r}=\frac{10 \times 7,8}{R} \quad \text { d'ou }: R=78 r
$$

Si on fait : $r=\mathrm{I}_{\mathrm{m}} 50$, on aura $R=\mathrm{I}_{2} 5$ mètres.

Donc, si on disposait les so prises prévues sur une circonférence de $250 \mathrm{~m}$. de diamètre, on aurait encore une surtension de so pour 100, soit 4 kilowatt de plus comme perte d'énergie.

Reprenons la formule: $V=\frac{I}{2 \pi C n r}+\frac{(n-1) I}{2 C_{\pi n l R}}$

L'erreur commise en adoptant $\Sigma \frac{\mathrm{I}}{\rho}=\frac{(n-\mathrm{I})}{R}$ est négligeable lursque $n$ est compris entre i 5 et 20 . Comme nous ne voulons pas dépasser ce nombre d'électrodes, et qu'en dessous l'erreur donne un voltage plus tort que celui qu'on ne veut pas dépasser, nous maintiendrons cette formule pour les recherches.

$R$ sera généralement donné par l'emplacement dont on disposera. $V$ est donné par la perte consentie, et $I$ est connu. Ce que l'on aura à déterminer sera donc: soit $n$, soit $r$.

Par raison d'économie, on a intérêt à réduire $r$.
Prenons, par exemple, $V=100$ volts, $I=200$ aimpères, $R=50 \mathrm{~m} .=5000 \mathrm{~cm} ., r=\mathrm{r} \mathrm{m} 50=\mathrm{r} 60 \mathrm{~cm} ., \mathrm{C}=\frac{\mathrm{l}}{5000}$ microhm-centimètres.

De la formule (I) on tire :

$$
n=\frac{R L}{2 \pi C V R-I}\left(\frac{1}{r}-\frac{1}{R}\right)
$$

Ce qui donne $n=\mathrm{I} 4$ électrodes, au lieu de ro.

Si $R=10.000$, on trouve $: n=\mathrm{I} 2$ électrodes.

De la formule (I), on peut également tirer :

$$
r=\frac{R I}{2 \pi \operatorname{Cn} R V-(n-\mathrm{I}) !}
$$

et si on fait : $R=5000$ et $n=10$, on a, dans cecas, $r=I^{m} m_{0}$, puisque $\frac{(n-1)}{R_{m}}$ vaut $\frac{7,8}{R}$

Si $R=$ I oooo, on trouve : $r=\mathrm{Im} 86$.

II. Electrodes disposées linéairement. - Examinons maintenant le cas où les'électrodes sont réparties uniformément le long d'une droite de longueur $L$.

Soient $n$ électrodes ainsi réparties; l'intervalle $d$ entre ceux électrodes consécutives sera $: d=\frac{L}{n-\mathrm{I}}$

Soient $A_{1} A_{2} A_{3} \ldots$, etc, , les charges prises par ces électrodes, et $r_{1} r_{2} r_{3} \ldots$, etc., leurs rayons respectifs.

Comme précédemment, si $r$ est petit devant $d$, on pourra considérer la surface équipotentielle comme étant une hémisphère, et, par conséquent, en prenant des électrodes hémisphériques, supposer la charge ramenée au centre.

Le potentiel à la surface de l'électrode de rang $p$ est :

$$
V_{p}=\frac{A_{p}}{r_{p}}+\Sigma_{p} \frac{A}{p}
$$

Comme, par définition, les électrodes sont toutes au même potentiel, $V$ est une constante qui est déterminée par la perte d'énergie consentie.

Dans cette équation, comme on ne peut pas prendre une valeur moyenne pour les différents points qui ne sont pas disposés d'une façon uniforme les uns par rapport aux autres, comme dans le premier cas, on est obligé de calculer $\Sigma_{p} \frac{A}{p}$ dans chaque cas.

Nous avons vu que le champ et l'intensité émis par un point étaient :

$$
H=-\frac{\mathrm{d} V}{\mathrm{~d} r}=\frac{A}{r^{2}} \quad \text { et } \quad I=C S H=2 \pi C A
$$

Nous pourrons donc toujours considérer que le courant émis par une électrode est directement proportionnel à la charge prise par cette électrode.

Ceci posé, reprenons la formule (2)

On peut prendre $A_{p}$ quelconque, et en déduire $r_{p}$ ou inversement. Nous n'examinerons que deux cas :

Io Nous prendrons $A$ constant, c'est-á-dire que nous ferons débiter à toutes les électroles la même intensité, et nous chercherons quel devra être son rayon pour que sa surface soit équipotentielle; 
$2^{0}$. Nous prendrons $r$ constant, c'est-à-dire que nous ferons des électrodes toutes identiques, et nous chercherons quelle doit être la charge de chacune d'elles pour que les surfaces soient toutes au même potentiel, et que l'intensité totale soit l'intensité voulue; puis, nous chercherons quel doit être le rayon commun, pour que le potentiel soit celui qu'on s'est fixé.

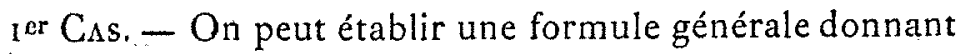
$\Sigma_{p} \frac{A}{p}$ en fonction de $r$ et $d$. De la formule générale on tire:

$$
r_{p}=\frac{A}{V-\Sigma_{p} \frac{A}{\rho}}
$$

Si nous supposons $n$ pair, en partaut de $p=o$ pour les électrodes les plus centrales, on a:

$\Sigma_{p} \cdot \frac{A}{p}=\left\{\begin{array}{c}\frac{2 A}{d}+\frac{2 A}{2 d}+\cdots+\frac{2 A}{\left(\frac{n}{2}-p-1\right) d}+\frac{A}{\left(\frac{n}{2}-p\right) d} \\ +\frac{A}{\left(\frac{n}{2}-p+\mathrm{I}\right) d}+\cdots+\frac{A}{\left(\frac{n}{2}+p\right) d}\end{array}\right\}$

La formule est légèrement différente si $n$ est impair. En tous cas, il est facile, puisqu'on doit toujours se donner $n$ et $L$. d"établir chaque fois l'équation de $\Sigma_{p} \frac{A}{\rho}$

Prenons par exemple $L=180 \mathrm{~m} .=18000 \mathrm{~cm} ., n=10$ on aura: $d=\frac{L}{n-1}=\frac{18000}{9}=2000 \mathrm{~cm}$, , ou 20 mètres .

$\begin{array}{llllllllll}A & A & A & A & A & A & A & A & A & A\end{array}$ $x-d-x-d-x-d-x-d-x-d-x-d-x-d-x-d-x-d-x$

$\begin{array}{llllllllll}r_{4} & r_{3} & r_{2} & r_{1} & r_{0} & r_{0} & r_{1} & r_{2} & r_{3} & r_{4}\end{array}$

Nous avons :

Fig. 2 .

$\Sigma_{0} \frac{A}{\rho}=\frac{2 A}{d}+\frac{2 A}{2 d}+\frac{2 A}{3 d}+\frac{2 A}{4 d}+\frac{A}{5 d}$ $=\frac{2 A}{d}\left(1+\frac{1}{2}+\frac{1}{3}+\frac{1}{4}+\frac{1}{10}\right)=2,1833 \frac{2 A}{d}$

$\Sigma_{1} \frac{A}{\mathrm{p}}=\frac{2 A}{d}\left(\mathrm{I}+\frac{\mathrm{1}}{2}+\frac{1}{3}+\frac{1}{8}+\frac{\mathrm{I}}{10}+\frac{1}{12}\right)=2,1416 \frac{2 A}{d}$

$\Sigma_{2} \frac{A}{\rho}=\frac{2 A}{d}\left(1+\frac{1}{2}+\frac{1}{6}+\frac{1}{8}+\frac{1}{\text { ro }}+\frac{1}{12}+\frac{1}{14}\right)$ $=2,0464 \frac{2 A}{d}$

$\Sigma_{3} \frac{A}{p}=\frac{2 A}{d}\left(1+\frac{1}{4}+\frac{1}{6}+\frac{1}{8}+\frac{1}{10}+\frac{1}{12}+\frac{1}{14}+\frac{1}{16}\right)$ $=1,8589 \frac{2 \mathrm{~A}}{\mathrm{~d}}$

$\Sigma_{4} \frac{A}{\mathrm{p}}=\frac{2 A}{\mathrm{~d}}\left(\frac{\mathrm{I}}{2}+\frac{\mathrm{I}}{4}+\frac{\mathrm{I}}{6}+\frac{\mathrm{I}}{8}+\frac{\mathrm{k}}{\mathrm{I} 0}+\frac{\mathrm{I}}{12}+\frac{\mathrm{I}}{14}+\frac{\mathrm{I}}{\mathrm{i} 6}+\frac{\mathrm{I}}{18}\right)$ $=\mathrm{I}, 4144 \frac{2 \mathrm{~A}}{d}$
On a pour chaque électrode :

$$
i=\frac{I}{n}=2 \pi C A \quad \text { d'où : } \quad A=\frac{I}{2 \pi C^{\top} n}
$$

Si l'on fait : $I=200, n=$ Io et $C=\frac{1}{5000}$, on a:

$$
A=\frac{10^{5}}{6,28}=15950 \quad \text { et } \quad \frac{2 A}{d}=\frac{10^{2}}{6,28}=15,95
$$

En prenant $V=100$, nous avons :

$$
\begin{array}{ll}
r_{0}=\frac{15950}{67,80}=236 \mathrm{~cm}=2^{\mathrm{m} 36} & \\
r_{1}=\frac{15950}{68,50} \text { soit } 2^{\mathrm{m} 33} \quad r_{2}=\frac{15950}{69,75} \text { soit } 2^{\mathrm{m}_{29}} \\
r_{3}=\frac{15950}{72,60} \text { soit } 2^{\mathrm{m} 20} \quad r_{4}=\frac{15950}{78,80} \text { soit } 2^{\mathrm{m}_{\mathrm{O} 2}}
\end{array}
$$

Pour comparer cette disposition rectiligne: avec la disposition circulaire, prenons :

$$
L=2 \pi R \times \frac{9}{10}
$$

Nous avions : $R=5 \mathrm{o}$. m., soit $2 \pi R=314 \mathrm{~m}$. et $d=3$ i mètres.

En prenant cette valeur $d=3 \mathrm{I}$ m., nous trouvons :

$$
\begin{array}{lll}
\frac{2 A}{d}=9,5 & \text { et } & r_{0}=\mathrm{I} \mathrm{m} 86 \\
r_{1}={ }_{\mathrm{I}}^{\mathrm{m} 85} & & r_{2}=\mathrm{I}_{\mathrm{I}} 83 \\
r_{3}=\mathrm{I}^{\mathrm{m}} 79 & & r_{4}=\mathrm{I}^{\mathrm{m}} 70
\end{array}
$$

Dans le cas de la disposition circulaire, on avait $r=\mathrm{I}^{\mathrm{m}} 90$.

A dévéloppement égal, la forme linéaire est done un peu plus avantageuse.

On voit, d'autre part, que plus $L$ est grand, plus les variations de $r$ sont faibles.

$\mathrm{Si}$ on avait $L=90 \mathrm{~m}$., et $d=\mathrm{ro}$,

On aurait :

$$
\begin{aligned}
& \frac{2 A}{d}=29 ; 50 \quad \text { et } \quad r_{0}=4^{\mathrm{m}} 7^{\circ} \\
& r_{1}=4^{\mathrm{m} 27} \quad r_{2}=3 \mathrm{~m} 68 \\
& r_{3}=3^{\mathrm{m}_{2} 7} \quad r_{4}=2^{\mathrm{m} 55}
\end{aligned}
$$

${ }_{2} \dot{\mathrm{e}}$ CAs. - Ce cas peut être ramené au précédent, lorsque $d$ est très grand devant $r$.

De la formule générale on tire : $A_{p}=r\left(V-\Sigma_{p} \frac{A}{\rho}\right)$

Etablissons $\Sigma_{p} \frac{A}{p}$ pour chaque point, il vient:

$\Sigma_{0:} \frac{A}{p}=\frac{A_{0}+A_{1}}{d}+\frac{A_{1}+A_{2}}{2 d}+\frac{A_{2}+A_{3}}{3 d}+\frac{A_{3}+A_{4}}{4 d}+\frac{A_{4}}{5 d}$ $=V-\frac{A_{0}}{r}$

$\Sigma_{1} \frac{A}{\mathrm{p}}=\frac{A_{0}+A_{2}}{d}+\frac{A_{0}+A_{3}}{2 d}+\frac{A_{2}+A_{4}}{3 d}+\frac{A_{2}}{4 d}+\frac{A_{3}}{5 d}+\frac{A_{4}}{6 d}$ $=V-\frac{A_{1}}{r}$ 
$\Sigma_{2} \frac{A}{p}=\frac{A_{1}+A_{3}}{d}+\frac{A_{0}+A_{4}}{2 d}+\frac{A_{0}}{3 d}+\frac{A_{1}}{4 d}+\frac{A_{2}}{5 d}+\frac{A_{3}}{6 d}+\frac{A_{4}}{7 d}$

$$
=V-\frac{A_{2}}{r}
$$

On peut alors, en remplaçant $A_{p}$ par sa valeur, obtenir 5 équations qui, résolues, donneront $\Sigma_{0}, \Sigma_{1}, \Sigma_{2}, \Sigma_{3}$ et $\Sigma_{4}$ en fonctions de $V_{1}$, de $d$ et de $r$.

Ces calculs, très longs, ne présentent pas grand intérêt, et on est très suffisamment renseigné par la première hypothèse.

Dans tous ces calculs, nous avons supposé que les électrodes, formées de coke pilé humide, étaient d'une conductibilité parfaite. Il resterait à vérifier la conductibilité de cette matière, afin de se rendre compte de l'erreur que l'on peut commettre ainsi.

\section{P. FERrier,}

Ingénieur-Electricien

Ancien Elève de l'Ecole stupérieure d'Llectricité.

\section{LE TUNNEL DU SIMPLON}

\section{Sa Perforation, son Exploitation}

\section{I. - HISTORIQUE}

L'Italie se trouve séparée du reste de l'Europe par la chaîne des Alpes. Cette frontière naturelle, qui l'entoure au nord sur une longueur de plus de mille kilomètres, a, pen-

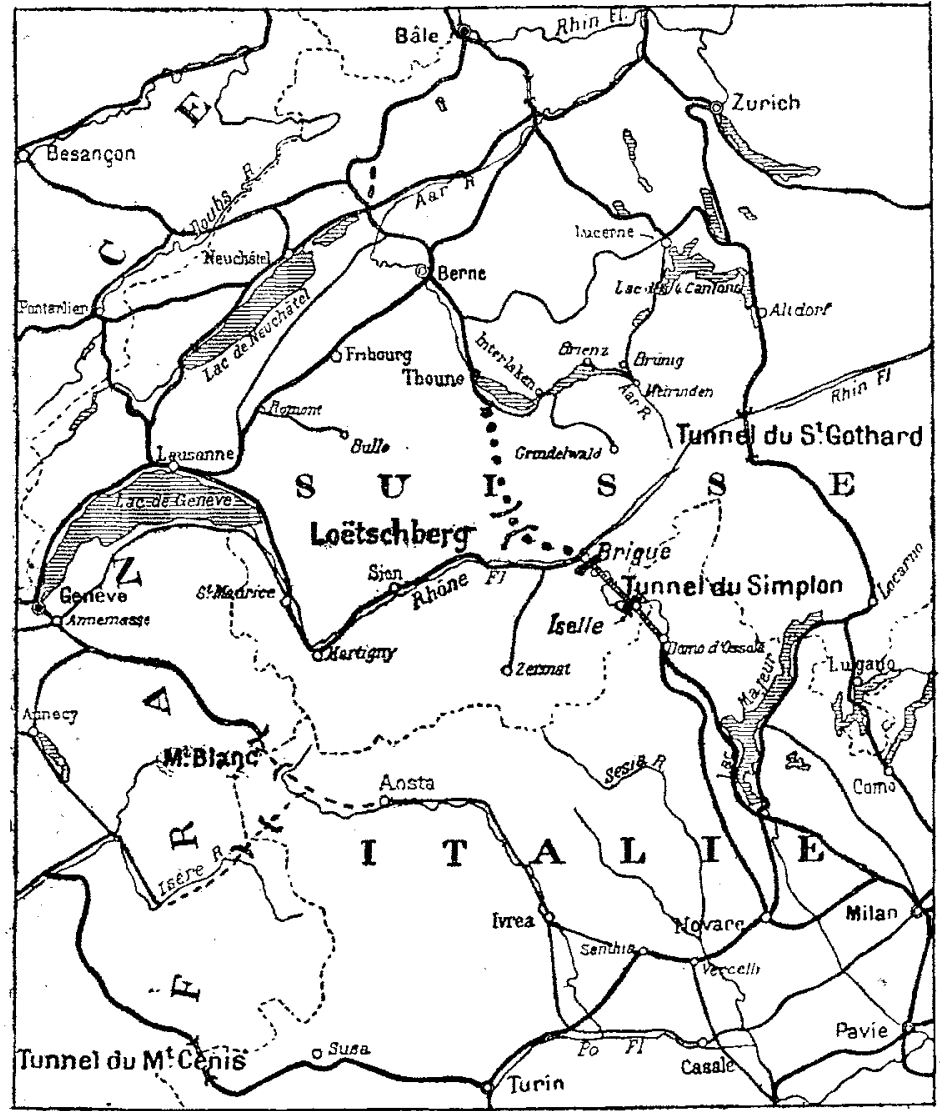

Fig. 1. - Carte des voies d'accès au simplon

dant longtemps, considérablement gêné son développement économique, et entravé ses relations avec ses voisins.
En 1800, Napoléon Ier ordonnait la construction dune route faisant communiquer les hautes vallées du Rhòne (en Suisse) et du Toce (en Italie), en passant par le col du Simplon, à l'altitude de 2000 mètres. Cette roule, dont la longueur est de 66,5 km., de Brigue à Domo d'Ossola, fut terminée en 1806 (juste cent ans avant la voie do fer), et elle resta, jusqu'à ces derniers temps, le seul passage important entre le Haut Valais et la Lombardie.

In 1871, le percement du Mont Cenis faisait communi. quer la France et le Piémont au moyen d'une voie ferrée, puis, en 1881 , le tunnel du Gothard mettait en relations rapides la Haute-Suisse et la Lombardie. Mais les voyageurs et les marchandises venant de l'Angleterle, de la Belgique, de la Hollande, uu de la région frontière francosuisse, devaient faire un long détour pour atteindre Gênes et Milan, les deux métropoles commerciales de l'Italie du

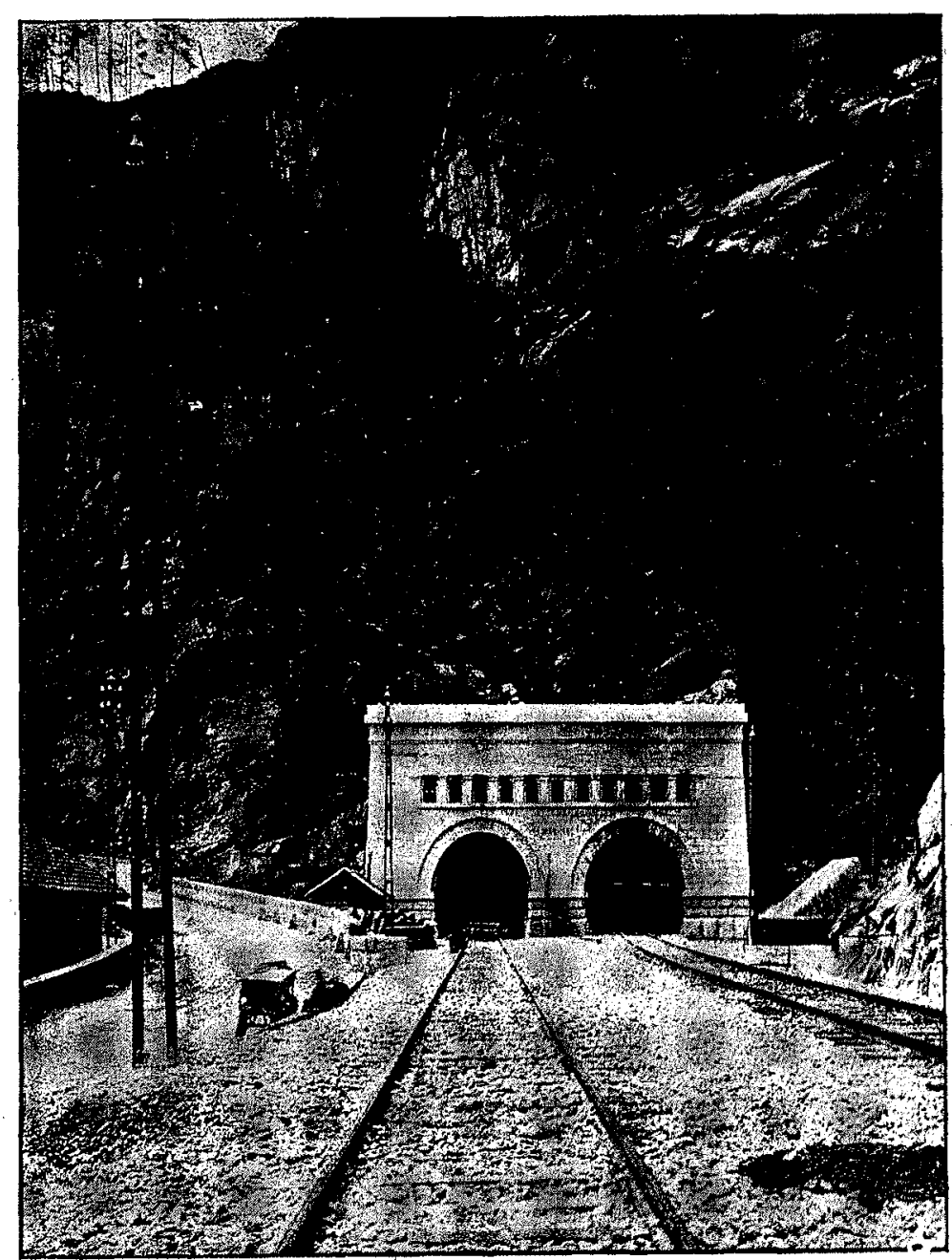

Fig. 2, - Vue de l'entríe du tunnel a Iselle

Nord. L'activité de plus en plus intense de lindustrie et du commerce modernes, ainsi que le besoin de plus en plus impérieux de franchir le plus rapilement possible les grandes distances, firent chercher une nouvelle voie intermédiaire entre le Mont-Cenis et le Gothard. Une ligne droite, tracée sur la carte de l'Europe, de Milan à Paris, ou à Calais, ou bien encore de Genes et Turin à Calais ou Anvers, coupant la chaine des Alpes dans le voisinage immédiat du Simplon, l'attention fut bientót portee de ce côté.

En 1856, le comte Adrien de la VALETTE obtint une concession pour la création d'une ligne de chemin de fer comprise entre le fort de l'Ecluse, près de Bellegarde (en France), et Arona, sur le lac Majeur (en Italie), par la Savoie, la haute vallée du Rhóne, le Simplon, le Val dOssola, et le lac Majeur. It constitua la Compagnie du 